\section{The Pentax-AWS is particularly suitable for facilitating intubation in edentulous geriatric patients}

\author{
Kunihiko Yamamoto, Akihiro Suzuki, Yuki Toyama, \\ Tomoki Sasakawa, Takayuki Kunisawa, Osamu Takahata, \\ and Hiroshi Iwasaki \\ Department of Anesthesiology and Critical Care Medicine, Asahi- \\ kawa Medical College, 2-1-1-1 Midorigaoka higashi, Asahikawa \\ 078-8510, Japan
}

To the editor: An edentulous state is commonly seen in the geriatric population. If a patient is edentulous and has sunken cheeks, mask ventilation is often difficult. On the other hand, intubation with the conventional Macintosh laryngoscope seems to be easier in edentulous patients because the laryngoscopist does not need to pay attention to protecting teeth from the solid device during direct laryngoscopy. The PentaxAWS system (AWS; HOYA, Tokyo, Japan) is a new indirect video laryngoscope with integrated tube guidance, which does not require direct line of sight during intubation. We evaluated whether dental status affected the intubation profile with the AWS in geriatric patients.

After obtaining institutional approval and written informed consent, we enrolled 40 patients aged more than 65 years scheduled for elective surgery. They were divided into two groups, the NT (normal teeth) group and the FD (full denture) group. Patients with partial lack of teeth were excluded from the study. In the FD group, all dentures were removed before anesthesia induction. Anesthesia was induced with propofol and fentanyl, followed by vecuronium administration. After complete paralysis occurred, confirmed by a peripheral nerve stimulator, intubation was performed with the AWS. A standard AWS technique with direct elevation of the epiglottis was used. The anatomical structure (epiglottis or vocal cord) seen on the monitor just after blade insertion and the rotation maneuver was documented. The time for intuba- tion, the percentage of glottic opening (POGO) score [1], the intubation difficulty scale (IDS) [2], and the number of intubation attempts were recorded. The time for intubation was defined as the time taken from the blade first passing the incisors until passage of the tracheal tube was completed.

Data were analyzed using the unpaired $t$-test and the $\chi^{2}$ test, and $P<0.05$ was considered significant.

Patient profiles were not different between the groups, except for the inter-incisor distance (IID). There was a significant difference between the groups in the anatomical structure observed after the initial blade rotation maneuver; the vocal cords were more frequently observed in the FD group. The total time for intubation was significantly less in the FD group than the NT group. The POGO score, IDS, and number of intubation attempts did not differ between the groups (Table 1).

Our result showed that intubation with the AWS was facilitated when the patients were edentulous. It seems that the increased IID in the edentulous patients may have been responsible for this result. As a consequence of the lack of teeth, the working space necessary for AWS manipulation was increased. This may have facilitated the insertion of the blade tip behind the epiglottis after blade rotation, resulting in faster laryngeal exposure and subsequent faster intubation.

\section{References}

1. Ochroch EA, Hollander JE, Kush S, Shofer FS, Levitan RM. Assessment of laryngeal view: percentage of glottic opening score vs Cormack and Lehane grading. Can J Anaesth. 1999;46:987-90.

2. Adnet F, Borron SW, Racine SX, Clemessy J, Fournier J, Plaisance $\mathrm{P}$, Lapandry C. The intubation difficulty scale (IDS): proposal and evaluation of a new score characterizing the complexity of endotracheal intubation. Anesthesiology. 1997;87:1290-7.

Address correspondence to: $\mathrm{K}$. Yamamoto

Received: November 28, 2008 / Accepted: February 27, 2009

Table 1. Demographic characteristics of patients

\begin{tabular}{lccc}
\hline & \multicolumn{2}{c}{ Results } \\
\cline { 2 - 4 } & Normal teeth group & Full denture group & Significance \\
\hline Age (years) & $77 \pm 6$ & $76 \pm 7$ & $\mathrm{NS}$ \\
Height (cm) & $159 \pm 6$ & $160 \pm 10$ & $\mathrm{NS}$ \\
Weight (kg) & $58 \pm 10$ & $59 \pm 12$ & $\mathrm{NS}$ \\
IID (cm) & $4.5 \pm 0.5$ & $5.8 \pm 1.1$ & $P<0.01$ \\
Anatomical structure (epiglottis/vocal cord) & $7 / 8$ & $12 \pm 4$ & $P<0.01$ \\
Time for intubation (sec) & $23 \pm 11$ & 87.6 & $\mathrm{NS}$ \\
POGO score & 83.5 & $(18 / 2 / 0)$ & $\mathrm{NS}$ \\
IDS (0/1/2) & $(17 / 2 / 1)$ & &
\end{tabular}

IID, inter-incisor distance; POGO, percentage of glottic opening; IDS, intubation difficulty scale 